\title{
The Importance of Meticulous Peri-operative Care in Managing High-Risk Patients Undergoing Bariatric Procedures
}

\section{Omar S Mansour*}

Consultant Colorectal and Laparoscopic General Surgeon, Assistant Professor and

Clinical Lecturer in General Surgery, Department of Surgery, School of Medicine, Al

Balqa Applied University, Al Salt, Jordan

*Corresponding Author: Omar S Mansour, Consultant Colorectal and Laparoscopic General Surgeon, Assistant Professor and Clinical Lecturer in General Surgery, Department of Surgery, School of Medicine, Al Balqa Applied University, Al Salt, Jordan.
Received: July 29, 2020

Published: September 30, 2020

(C) All rights are reserved by $\mathbf{O m a r} \mathbf{S}$

Mansour.

\begin{abstract}
Background: Recent advances in laparoscopic and endoscopic technology has led to simplify many bariatric procedures especially laparoscopic sleeve gastrectomy and endoscopic intragastric balloon insertion. Because of the recent high demand for bariatric procedures, there has been a recent trend to perform bariatric procedures by general surgeons and physicians who were not trained to perform such procedures. Many surgeons would refuse to perform bariatric procedures on high risk patients to avoid complications of these elective procedures.

Analysis: In this paper, two cohorts of high-risk patients were analyzed, laparoscopic sleeve gastrectomy and endoscopic intragastric balloon insertion groups. All of these procedures were done by the same surgeon and for the purpose of weight loss. All of the selected patients had significant comorbidities that could have led to increased morbidities.

Conclusion: High risk bariatric patients carry a significant peri operative risk. Proper preoperative optimization and meticulous perioperative care can reduce post-operative risks and avoid significant post-operative morbidities and potential mortalities. There were no mortalities or any ICU admissions for any of the high and low risk patients.
\end{abstract}

Keywords: Laparoscopic Sleeve Gastrectomy; Intragastric Balloon; Candida; High Risk Patients; Endoscopy; Perioperative

\section{Introduction}

Recent advances in laparoscopic and endoscopic technology has led to simplify many bariatric procedures especially laparoscopic sleeve gastrectomy and endoscopic intragastric balloon insertion. Because of the recent high demand for bariatric procedures, there has been a recent trend to perform bariatric procedures by general surgeons and physicians who were not trained to perform such procedures. Many surgeons refuse to perform any bariatric procedures on high risk patients to avoid complications of these elective procedures.
In this paper, two cohorts of patients were analyzed, laparoscopic sleeve gastrectomy and endoscopic intragastric balloon insertion. The first cohort is the surgical group and the second cohort is the endoscopic (non-surgical) group. All of these procedures were done for the purpose of weight loss. All of the selected patients in this study had significant comorbidities that could have led to increased morbidities. All of these procedures were performed by the same surgeon who is a western trained laparoscopic and endoscopic colorectal surgeon with extensive training and experience in complex upper endoscopic, laparoscopic and open gastrointestinal 
surgery. High risk patients were searched and selected retrospectively and their outcome was analyzed.

Four patients were found in the high-risk sleeve gastrectomy cohort and two patients were found in the high risk intragastric balloon group.

\section{Case Series}

The patients

The surgical group

In the surgical group, the first patient, who was a redo sleeve gastrectomy (done by another surgeon), was found postoperatively to be non-cooperative and alcoholic.

The second patient was diagnosed with severe metabolic syndrome few years pre-operatively. In addition, this second patient had Child's B liver cirrhosis and respiratory failure. He was using CPAP on a daily basis during sleep.

The third patient had a severe psychiatric background, with multiple surgical and medical diseases This female patient had severe epilepsy, narcolepsy, recent laparoscopic rectopexy and chronic constipation, chronic abdominal pain, nausea and vomiting.

The fourth patient was diagnosed with severe reflux diseases pre-operatively. A pre-operative upper GI endoscopy, done by the same surgeon showed severe reflux oesophagitis and Barrett's esophagus.

\section{The endoscopy (intragastric balloon) group}

In the Intragastric balloon cohort, two patients in the endoscopic cohort were found to be high risk.

The first patient had multiple comorbidities including liver cirrhosis and respiratory failure requiring daily CPAP usage during sleep.

The second case was a psychiatric female patient with barely controlled relapsing ulcerative pan-colitis and recurrent nausea.

\section{Early post-operative complications}

The sleeve gastrectomy group

The first patient, a 41 year old male patient, had a redo open sleeve gastrectomy and a very large incisional hernia repair. This patient, who was a redo sleeve gastrectomy (done by another sur- geon), was found postoperatively to be non-cooperative and alcoholic. This patient had a gastric band procedure followed 2 years later by laparoscopic sleeve gastrectomy by another bariatric surgeon. The patient failed to lose any weight but developed large symptomatic incisional hernia. An open incisional hernia repair combined with open sleeve gastrectomy was done at our centre. The patient developed vomiting on the third day post operatively. He then started to develop fever two weeks later. He was treated conservatively with IV antibiotics. The patient was found to be an alcoholic and didn't follow the post-operative oral intake instructions. Against the dietician's advice, he started solid diet less than one week post operatively. The vomiting kept on recurring and an upper GI endoscopy was done that showed narrowing of the gastric sleeve at the antral area. Pneumatic dilatation was performed and this procedure went smoothly without any complications. This procedure was done twice. An abdominal CT scan was done later because of the recurred vomiting and the ongoing fever. An intra-abdominal collection was found on the CT just anterior to the stomach. It was drained under ultrasound guidance. Culture and sensitivity showed heavy Candida growth. Intra-abdominal Candida infection was diagnosed, probably secondary to minor gastric leakage. This was treated with percutaneous drain insertion and regular wash through the drain using oxidized water solution. A revision open abdominal surgery was done two months after the sleeve gastrectomy to perform definitive drainage, debridement of the infection and a pyloroplasty. The patient recovered quite well and lost $49 \%$ of total body weight (73 Kgs).

The second patient, a 43 year old male, was diagnosed with severe metabolic syndrome few years pre-operatively. In addition, this second patient had Child's B liver cirrhosis and respiratory failure. He was using CPAP on a daily basis during sleep. He also suffered from poorly controlled both diabetes mellitus and hypertension. The laparoscopic sleeve gastrectomy was done two weeks post removal of an intragastric balloon. The procedure was not straight forward due to heavy adhesions from previous open cholecystectomy and cirrhotic liver. The patient had a drain inserted intraoperatively and left for 10 days post op. The drain was filling with $200-300 \mathrm{ml}$ of blood on a daily basis for one week. It dried around 8 days post operatively. The patient was transfused with 5 units of packed RBC's. He recovered quite well and lost $20 \%$ of TBW (21 Kgs). His blood pressure, diabetes mellitus and the sleep apnea all improved dramatically and the patient stopped using his CPAP machine. 
The third patient, a 37 year old female, had a severe psychiatric background, with multiple surgical and medical diseases This female patient had severe epilepsy, narcolepsy, recent laparoscopic rectopexy and chronic constipation, nausea and vomiting. This patient had a combined laparoscopic sleeve gastrectomy and a laparoscopic cholecystectomy at the same time. Progressive loss of appetite and recurrent vomiting started to took place 6 months post operatively. Minor non obstructive twisting of the gastric conduit was seen during an upper GI endoscopy done by the same surgeon six months post op, probably secondary to adhesions. The severe nausea was treated conservatively. She lost $35 \mathrm{Kgs}$ post op $(33 \%$ of TBW).

The fourth patient, a 33 year old female, was diagnosed with severe reflux diseases pre-operatively. A pre-operative upper GI endoscopy, done by the same surgeon showed Barret's oesophagitis. She was strongly advised against the sleeve gastrectomy but she insisted on having the procedure done. The procedure went smoothly without any perioperative complications. The patient did have mild reflux post operatively for 3 months but disappeared 4 months post op. Postoperative routine follow up OGD showed that the reflux oesophagitis disappeared and repeat biopsies of the gastro-oesophageal junction confirmed the disappearance of Barrett's esophagus. She lost 19 Kgs 16 months post op (25\% of TBW).

\section{The endoscopy (intragastric balloon) group}

The first patient, a 43 year old male, had multiple comorbidities including liver cirrhosis and respiratory failure requiring daily CPAP usage during sleep. The procedure was well tolerated and did not have any complications and the balloon was removed six months post op. The patient lost around $10 \mathrm{Kgs}$ of weight $(9.5 \%$ of total weight). This procedure was done in preparation for sleeve gastrectomy which done seven months after the insertion (4 weeks post removal of the balloon).

The second patient, a 35 year old female, was a psychiatric female patient with poorly controlled relapsing ulcerative pan-colitis. She had a Spatz 12 months intragastric balloon. The patient was on mesalazine, steroids and multiple neuroleptic medications. The patient requested removal of the intra gastric balloon 33 days post insertion due to severe side effects including nausea, vomiting and hematemesis. She lost $12 \mathrm{Kgs}$ during that period $(14.5 \%$ of total weight).

\section{Discussion}

The outcome of bariatric procedures depends on many factors including surgeon's skills and experience, patient's adherence to strict low-calorie dieting and procedure type.

In the laparoscopic sleeve gastrectomy patients, the average the patient loses around $60 \%$ of their excess weight.

On the other hand, the endoscopic (non-surgical) intra gastric balloon insertion, is a temporary procedure that allows the patient to lose around 30 to $47 \%$ of their excess body weight.

Intragastric balloon placement has been proven as a safe, relatively low-cost and well-tolerated minimally invasive procedure for weight loss.

In a study done by Singh., et al. and his colleagues, they investigated weight loss 18 and 24 months after intragastric balloon insertion. The weight loss was significantly decreased at 18 or 24 months compared to 6 months post insertion indicating weight regain after intragastric balloon removal [1]. The average weight loss after intragastric balloon insertion were significantly decreased at 18 or 24 months compared to 6 months post operatively. It can be concluded that weight gain does happen after balloon removal. Serious complications were observed in $<5 \%$ for this procedure [1].

This would give encouraging results only if the overweight patients enroll in a strict and medically supervised weight loss program.

Complications rate after laparoscopic sleeve gastrectomy ranges from 2.9 to $13.5 \%$ [2].

After laparoscopic sleeve gastrectomy, the average leak is estimated to be around $2.8 \%$. Many studies suggest that most leaks can be managed conservatively. The use of staple-line reinforcement was not proven to decrease leakage form the staple line post operatively [3].

In a study that investigated the feasibility of performing sleeve gastrectomy immediately after removing the intragastric balloon on the same day, there were no immediate post-operative complications in this group. But the number of patients who had immediate laparoscopic sleeve gastrectomy post balloon removal was not significant [4].

In a study that investigated the importance of positive fungal culture in post bariatric surgery peritonitis, there was a significant number of patients who were Candida positive on peritoneal fluid culture and analysis [5]. There was a significant number of fluco- 
nazole resistant fungal strains on peritoneal fluid cultures results.

A recent paper from Turkey, a cohort of patients underwent concomitant laparoscopic cholecystectomy and laparoscopic sleeve gastrectomy. It was compared to another cohort who had laparoscopic sleeve gastrectomy alone. No significant differences were seen between the rate of complications between the sleeve gastrectomy patients and the combined procedure patients [6].

\section{Conclusion}

Surgical and endoscopic bariatric procedures have both been proven to be safe and effective in weight reduction. In high risk pa-

\section{Bibliography}

1. Shailendra Singh., et al. "Intragastric Balloon Versus Endoscopic Sleeve Gastroplasty for the Treatment of Obesity: a Systematic Review and Meta-analysis". Obesity Surgery 30.8 (2020): 3010-3029.

2. Bellorin O., et al. "Laparoscopic conversion of sleeve gastrectomy to Roux-en-Y gastric bypass for acute gastric outlet obstruction after laparoscopic sleeve gastrectomy for morbid obesity". Surgery for Obesity and Related Diseases 6.5 (2010): 566-568.

3. Alexander Aurora., et al. "Meta Analysis of leak after laparoscopic sleeve gastrectomy for morbid obesity". SAGES, Abstracts (2011).

4. Naif Al Enazi., et al. "Laparoscopic sleeve gastrectomy following removal of intragastric balloon on the same time, is it feasible?" SAGES abstracts (2011).

5. N Zappella., et al. "Positive peritoneal fluid fungal cultures in postoperative peritonitis after bariatric surgery". Clinical Microbiology and Infection 21.9 (2015): 853.e1-853.e3.

6. Mürşit Dincer and Fadli Dogan. "The effect of concomitant cholecystectomy and sleeve gastrectomy on morbidity in highrisk obese patients with symptomatic gallstones". Wideochir Inne Tech Maloinwazyjne 14.2 (2019): 237-241.

\section{Assets from publication with us}

- Prompt Acknowledgement after receiving the article

- Thorough Double blinded peer review

- Rapid Publication

- Issue of Publication Certificate

- High visibility of your Published work

Website: www.actascientific.com/

Submit Article: www.actascientific.com/submission.php

Email us: editor@actascientific.com

Contact us: +919182824667 